Cinémas

Revue d'études cinématographiques

Journal of Film Studies

\title{
Cinéma structurel et musique répétitive : correspondances
}

\section{Luc Desjardins}

Volume 9, numéro 2-3, printemps 1999

Les Scénarios fictifs

URI : https://id.erudit.org/iderudit/024795ar

DOI : https://doi.org/10.7202/024795ar

Aller au sommaire du numéro

Éditeur(s)

Cinémas

ISSN

1181-6945 (imprimé)

1705-6500 (numérique)

Découvrir la revue

Citer cet article

Desjardins, L. (1999). Cinéma structurel et musique répétitive :

correspondances. Cinémas, 9(2-3), 219-231. https://doi.org/10.7202/024795ar
Résumé de l'article

Cet article examine les différentes solutions apportées par les cinéastes " structurels " et les compositeurs " répétitifs " américains à ce problème formel fondamental commun à la musique et au cinéma : l'organisation du temps. Après avoir circonscrit historiquement et géographiquement ces deux tendances, l'auteur décrit les principales caractéristiques formelles qui leur sont communes, puis dégage brièvement les modifications qu'elles apportent à la relation spectateur / oeuvre. 


\title{
Cinéma structurel et musique répétitive: correspondances
}

\section{Luc Desjardins}

\begin{abstract}
RÉSUMÉ
Cet article examine les différentes solutions apportées par les cinéastes "structurels" et les compositeurs "répétitifs" américains à ce problème formel fondamental commun à la musique et au cinéma : l'organisation du temps. Après avoir circonscrit historiquement et géographiquement ces deux tendances, l'auteur décrit les principales caractéristiques formelles qui leur sont communes, puis dégage brièvement les modifications qu'elles apportent à la relation spectateur / œuvre.
\end{abstract}

\begin{abstract}
The article examines the different solutions structural filmmakers and the American repetitive composers brought to a fundamental formal problem common to music and cinema: the organization of time. After historically and geographically delineating these two tendencies, the author first describes their shared principal formal characteristics, then briefly outlines the modifications that these processes bring to the relationship between spectator and work, thereby redefining, the mode of reception.
\end{abstract}

Formellement le cinéma est un art du mouvement se concrétisant par le déroulement dans le temps d'éléments sonores et visuels. [...] Le problème formel fondamental qui se pose à la réalisation d'un film est un problème d'organisation du temps. [...] À un degré d'abstraction très élevé, les problèmes du cinéma sont 
les mêmes que ceux de la musique, problèmes de rythme, problèmes d'orchestration de différents thèmes visuels, problèmes de développement de ces thèmes dans le temps suivant une logique interne (Hébert, p. 10).

Les différentes solutions apportées par les cinéastes et les compositeurs à ce problème formel nous permettent de mettre en relief plusieurs correspondances entre ces deux disciplines artistiques. Nous nous attacherons plus particulièrement à l'étude de deux tendances parmi les mouvements d'avant-garde cinématographiques et musicaux : le cinéma structurel et la musique répétitive américaine. Il ne sera donc pas question ici de déterminer ce qui, du cinéma ou de la musique, a inspiré l'autre; il s'agira plutôt d'établir des rapports d'analogie et d'approfondir un domaine qui n'est généralement qu'esquissé dans la littérature.

Au-delà des querelles lexicologiques à propos du caractère approprié ou non de l'étiquette "répétitive» ou "minimale» appliquée aux cuvres de Philip Glass, Steve Reich, Terry Riley et La Monte Young ', une chose est sûre: on dénombre suffisamment de caractéristiques communes pour permettre de les regrouper en un courant musical spécifique (nous les appellerons dorénavant les "répétitifs américains"). À cet égard, il est symptomatique de constater qu'un débat presque identique eut lieu autour de l'expression "cinéma structurel" proposée par Sitney ${ }^{2}$.

Le courant musical des répétitifs américains possède un pôle géographique: la ville de New York. C'est là que les compositeurs cristallisèrent individuellement, et parfois collectivement, les fondements de leurs démarches propres: Terry Riley fait partie du Theater of Eternal Music (fondé en 1964) de La Monte Young; Steve Reich et Philip Glass jouent tous les deux dans leurs ensembles respectifs (au moins au début) et font partie de programmes communs lors de tournées européennes.

C'est aussi à New York que des liens se tissent entre les deux microcosmes créateurs de la musique et du cinéma d'avant-garde: on trouve dans cette ville le compositeur et cinéaste Tony Conrad, fréquemment laissé pour compte par les commentateurs de ce club 


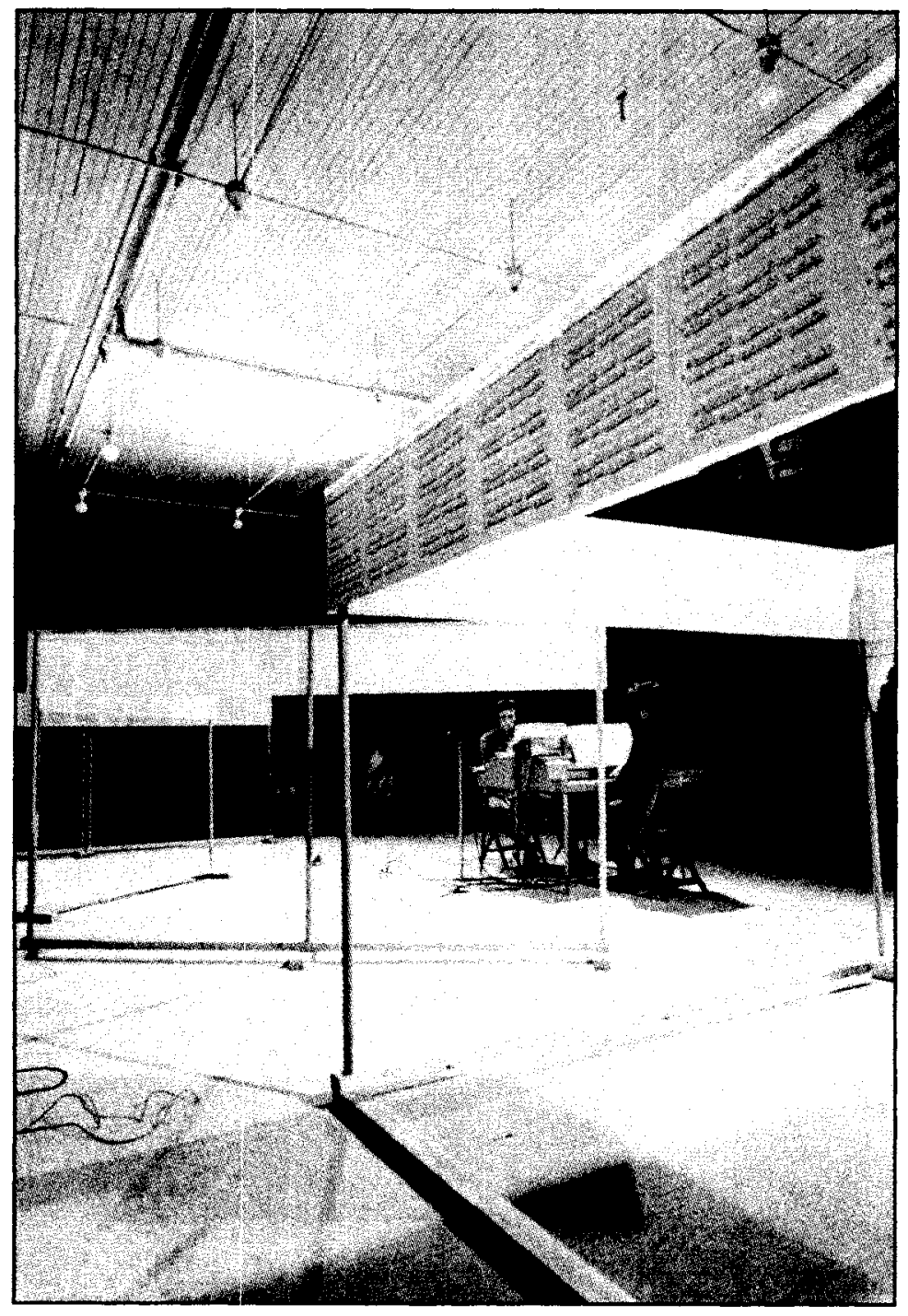

\section{Philip Gilass et Steve Reich en concert le 19 mai 1968}

sélect (il était pourtant lui aussi membre du groupe de La Monte Young, mais son ceuvre est moins imposante quantitativement ${ }^{3}$ ) et le groupe pluridisciplinaire Fluxus, important lieu de rencontre entre cinéastes et musiciens (Yoko Ono, John Cale, etc. ${ }^{4}$ ). Pour la petite histoire, mentionnons que Glass, Reich, Riley et La Monte 
Young sont tous nés autour des années 1935-1936; que les compositions qui feront l'objet de notre étude sont apparues entre 1964 (In C de Riley et The Well-Tempered Piano de La Monte Young) et 1976 (Music for 18 Musicians de Reich et Einstein on the Beach de Glass), recouvrant ainsi les grandes années du cinéma structurel, de 1967 à 1974 (James, p. 263).

Les compositions des répétitifs américains se démarquent à plusieurs égards des canons de la musique occidentale, contemporaine ou d'avant-garde. Il s'agit d'une musique fondamentalement réflexive de par sa technique compositionnelle et structurelle privilégiée: l'énoncé répétitif. Cet énoncé prend différentes formes selon le compositeur: «[...] additive process, in which a simple melodic figure is altered after a number of repetitions by the addition or subtraction of one or a group of related notes" chez Glass (Glass, 1974); "déphasage graduel de deux ou plusieurs séries d'un même motif répété jouées simultanément" chez Reich"; "[...] a solo improviser who "multiplies himself in performance by means of repetition, tape loops, tape delay systems and multi-tracking devices" chez Riley (Nyman, 1974 , p. 123); ou encore, "[...] prolongation ininterrompue d'un son isolé et soigneusement choisi chez La Monte Young" (Caux, 1977, p. 85).

On voit poindre sous ces procédés l'influence séminale de John Cage. On peut y relever ce que Nyman définit comme le principe de structure rythmique (dans la continuité des travaux de Satie et Webern), le plus souvent appliqué à des gammes limitées et fixées (Nyman, 1975); et surtout le principe d'indétermination: "Indeterminacy implies art as process. No beginning, no middle, no end; that is, no longer will "objects" of music exist in that sense, but each new performance, each new circumstance will create a continually variable process of ideas" (Cope, p. 169).

Si l'influence de Cage sur la technique compositionnelle privilégiée des répétitifs américains est présente et indéniable, elle n'est toutefois pas inéluctable. Ainsi, au principe d'indétermination prônant l'acceptation de tous les sons quels qu'ils soient, les répétitifs américains substituent la prolongation fascinante de certains sons rigoureusement déterminés, ou leur répétition sur une longue durée. Il est question ici de redécouvrir les 
fondements du son proprement dit (hauteur, durée et silence) plutôt que de suivre une pensée d'ordre plus ou moins narrative ou téléologique (comme dans la musique baroque, classique, romantique ou même moderne).

De plus, la plupart de ces compositions proposent une musique volontairement tonale et / ou modale, de structure rythmique très simple, le plus souvent cyclique, se démarquant ainsi de John Cage, mais aussi du système sériel ("[...] la tendance "électronique sérielle" représentait la tradition» dominante en musique électro-acoustique aux États-Unis) (Chion et Reibel, p. 180). Par exemple, selon La Monte Young: «[...] the serialist dealt with structure as sequence, rather than in my music where I am interested in structures as simultaneity" (cité dans Wentz, p. 48). On peut également y voir une influence déterminante des musiques non occidentales (indiennes chez Glass, Riley et La Monte Young; africaines chez Reich) "[...] fondées sur le rythme tandis que les musiques populaires occidentales sont fondées sur l'harmonie» (Tordjman, p. 100).

Contrairement à d'autres démarches caractérisées par l'accumulation des médias audiovisuels, Riley et consorts se concentrent sur un propos musical précis, préexistant, qui met les techniques et les genres à son service, au lieu d'évoluer vers une conception "événementielle». Conséquemment, ces répétitifs américains n'utilisent les moyens électro-acoustiques «de pointe" que pour leurs pouvoirs très simples qu'ils mettent au service de leur démarche musicale, évitant ainsi une sophistication ou une particularisation.

Ces avant-gardes musicale et filmique ne se rejoignirent pas simplement sur un plan théorique, mais aussi sur le terrain. La musique des répétitifs américains, très peu jouée dans les salles de concert de musique contemporaine, s'est développée de façon marginale: dans les circuits parallèles, les universités, les musées, les galeries d'art, etc., précisément là où les cinéastes structurels eux-mêmes tentaient d'imposer à leurs films le titre d'objet d'art. Glass décrit bien la teneur de savoureuses séances mixtes:

In fact, there was a period in which I'd show a film of Michael's [Snow] in the first half of the concert and I'd play in the second half. We showed either Wavelength 
or Back and Forth. What linked us together was a preoccupation with process. [...] In the early 70's about a third of the audience would walk out during the film and another third would walk out during the music. We'd have a handful of people by the end of the concert (I didn't mind that) (Simon, p. 137).

À un niveau un peu moins prosaïque, Noguez établit aussi des rapprochements :

Le film improprement dit "structurel " apparaît comme la trace particulière dans le cinéma underground américain d'une mutation qui se préparait dans d'autres aires du cinéma expérimental international et qui est liée, d'ailleurs, à l'évolution d'autres arts (1985, p. 350).

Pour les fins de notre étude, nous nous en tiendrons aux critères énoncés par Sitney, sans nous formaliser outre mesure des querelles de chapelles entourant le flottement sémantique de la notion de "cinéma structurel". Pour Sitney, les quatre traits caractérisant le film structurel sont:

[...] l'immobilité de la caméra (souvent dilatée par d'invisibles répétitions en boucles), les effets stroboscopiques (ou de clignotement), la répétition exacte et consécutive d'un même plan ou d'une série de mêmes plans (films à boucles visibles) et finalement le réenregistrement d'images projetées sur écran (refilmage) (Noguez, 1985, p. 347-348).

On a donc affaire à des propositions cinématographiques rigoureusement ordonnées autour d'un nombre restreint de codes filmiques. La palette de ces combinaisons d'un nombre limité de paramètres s'étend de la synchronisation jusqu'à la juxtaposition arbitraire.

Nous sommes en présence d'une évolution très proche de celle des répétitifs américains: un retour conscient aux origines, "[...] the pursuit of the formal logic of the medium itself" (James, p. 267). Le film devient donc une structure réflexive, concernée par sa propre nature et ses propres capacités de signification, et implicitement critique de l'illusionnisme narratif qui lui fut imposé historiquement. 
Après avoir dégagé de la démarche créatrice de ces deux mouvements certaines correspondances intimes, examinons brièvement certains films structurels pour vérifier en quoi ils correspondent bien au modèle proposé par Sitney, tout en proposant des rapprochements avec quelques compositions des répétitifs américains. Cela nous permettra d'évaluer dans quelle mesure ces œuvres, au niveau de leur réception, impliquent une modification des habitudes des spectateurs (voire même de leurs aptitudes!).

Commençons par examiner Fracture (David Rimmer, 1973). Ce film muet, constitué de 19 plans, est en fait une dissection de deux fragments reproduits par impression optique et montés en parallèle selon la forme A / B / A' / B' / A" / B" / etc. Aucun de ces plans n'est tout à fait identique: le traitement à la tireuse optique permet au cinéaste de faire varier, pour chacun des deux fragments, un ou plusieurs paramètres spatiaux ou temporels (l'échelle des plans, le cadrage, la durée, même le sens de déroulement de l'image). Ces variations, par un jeu sur les conventions narratives (champ / hors-champ, absence de résolution), forcent une remise en question de notre attitude spectatorielle. À cet égard, le douzième plan du film est peut-être le plus décisif. Ce douzième plan est une reprise du plan initial, mais dont le déroulement est inversé. Cette simple inversion bouleverse totalement notre interprétation des relations entre les 11 premiers plans et mine par le doute notre expectative des plans suivants.

On peut associer ce film à plusieurs des premières compositions de Philip Glass, notamment One + One (1968), une pièce pour percussions, avec les doigts, sur table munie d'amplificateurs. La partition est une combinaison continue de deux unités rythmiques simples (exemple de combinaison: A B 2B A 3B A 2B A B, etc. ${ }^{6}$. Ces deux ouvres proposent intrinsèquement une redéfinition de leur réception par la mise en place d'une structure relativement simple basée sur la répétition d'un nombre restreint de paramètres (deux fragments filmiques et deux blocs rythmiques).

Passons maintenant au film d'un autre cinéaste canadien ${ }^{7}$, Pas de deux de Norman McLaren (1967). Ce film exploite 
intensivement le quatrième critère proposé par Sitney, le procédé de réenregistrement d'images:

[...] une surimpression de chaque prise de vue sur ellemême environ 10 fois. Mais entre chacune des opérations, nous avons établi un décalage de cinq photogrammes. Le résultat donna 10 impressions de la même image (et du même mouvement), chacune avec un décalage d'un quart de seconde (McLaren, cité dans Bonneville, p. 83).

Le caractère réflexif du dédoublement, de la surmultiplication des mouvements combinés à l'immobilisme de la photographie, évoque certaines compositions de Terry Riley, Poppy Nogood ér the Phantom Band (1967) par exemple, où le principe de base est la lente et presque insensible transformation de brefs motifs de base de telle manière que l'on obtient une sorte de masse mobile, tout en donnant l'impression d'immobilité.

Cela dit, venons-en maintenant à ces fameux New-Yorkais. D'abord Andy Warhol qui, avec Empire (1964), semble appliquer au cinéma les expériences sur la périodicité d'un son continu amorçées par La Monte Young en 1960. Ce film muet exploite jusqu'à son paroxysme le premier critère de Sitney, l'immobilité de la caméra: «[...] les huit heures [du film] sont le bout à bout, sans montage, des bobines successives tournées en plan fixe avec une caméra [...] braquée sur l'Empire State Building [...]" (Noguez, 1985, p. 265). On a ici une correspondance parfaite avec la Drift Study (La Monte Young, 1966) : un son unique formé de fréquences électroniques absolument stables.

Plutôt que d'un mode de réception ludique, tel que proposé par Fracture, c'est peut être ici d'un mode de confrontation qu'il faudrait parler! Stephen Koch a bien décrit le caractère hermétique de ce film-épreuve:

Certains événements, cependant, ont réellement lieu dans Empire. Le soleil se déplace dans le ciel. Et les projecteurs qui illuminent les 30 derniers étages de l'édifice s'allument au crépuscule. Mais, le spectateur doit être très attentif: ces moments d'extase sont brefs et presque imperceptibles (p. 84). 


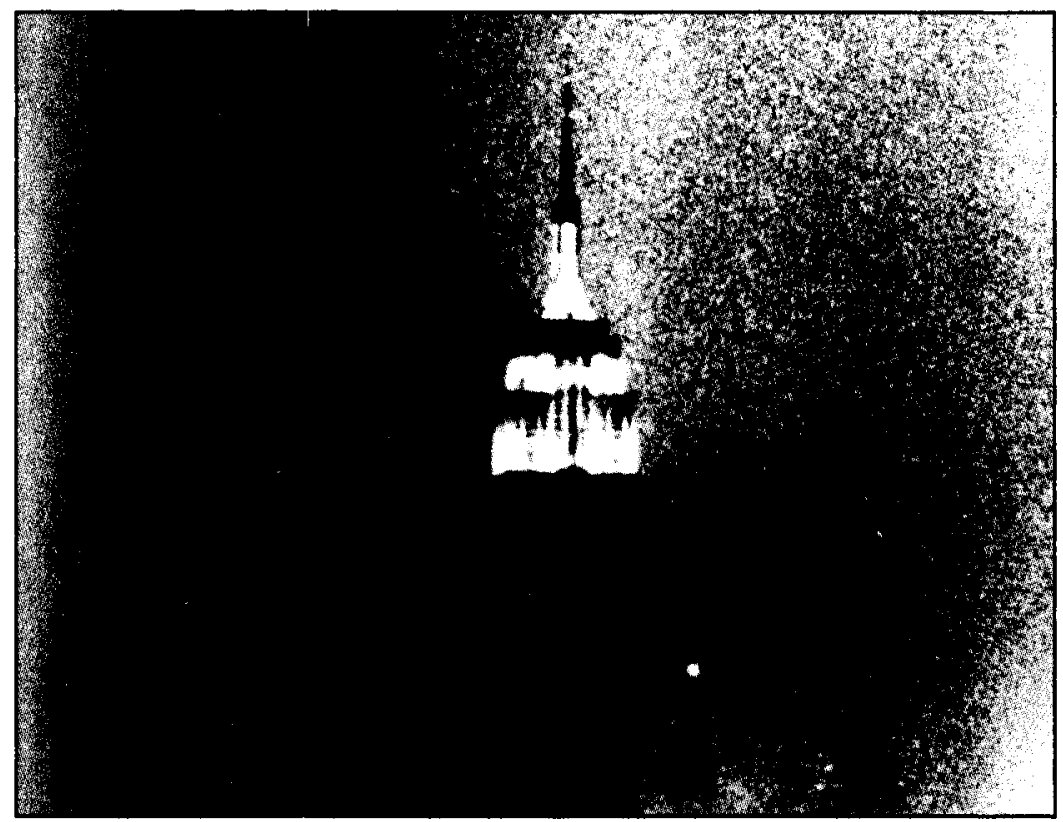

\section{Empire d'Andy Warhol (1965)}

On retrouve également une similarité frappante entre (nostalgia) (Hollis Frampton, 1971) et certaines pièces de Steve Reich, entre autres Phase Patterns (1970), basées sur le déphasage de figures élémentaires. Dans ce film (premier d'une série de sept, rassemblés sous le titre Hapax Legomena), Frampton utilise lui aussi la caméra immobile pour jouer sur le décalage images / sons (par synchronisme retardé, si on peut dire), inscrit dans une structure répétitive.

Le premier plan du film nous présente une pièce encombrée. Le narrateur pince-sans-rire décrit avec précision une photographie censée représenter un portrait et les circonstances ayant entouré sa réalisation. A un moment, la décomposition de l'image fait apparaître de légers indices qui permettent au spectateur de comprendre que l'objet filmé est en fait une photographie qui, posée sur un élément chauffant, se consume progressivement. Après la combustion complète de la photo, un deuxième plan nous présente ce que l'on devine être la photo que le narrateur décrivait au plan précédent, alors que 
simultanément, on l'entend décrire ce que l'on suppose maintenant être la photo que l'on verra au plan suivant. Et ainsi de suite, jusqu'au dernier plan, où le narrateur décrit une image que l'on ne verra pas.

Tout au long du film, le spectateur est convié ironiquement, par des expressions du narrateur ("as you can see", "if you look closely", "Look at it! Do you see what I see!»), à regarder une image qui vient de lui être décrite, mais qu'il ne verra plus. Et, comme si cela ne suffisait pas, ces photos ont souvent été choisies parmi une série de négatifs ou d'épreuves d'une même séance, que l'on ne verra naturellement pas ( I I wish you could have seen them [...]", ironise le narrateur). Ici, le mode de réception ludique proposé par Fracture est augmenté d'un autre paramètre: la bande sonore, qui vient complexifier encore le jeu de confrontation entre plans consécutifs.

On propose souvent, à la fois dans les comptes rendus critiques et dans les articles écrits par les cinéastes eux-mêmes, des lectures quasi mystiques de leur plan transcendantal et de leur effet hypnotique sur le spectateur (James, p. $260^{8}$ ). Sans contester la valeur de ces interprétations, je préfere mettre l'accent sur le caractère fondamentalement réflexif commun à toutes les œuvres examinées précédemment. Tous les exemples cités procèdent d'une échelle temporelle non narrative (film ou musique perçue comme "présence»; dénuée de structure dramatique, même réflexive; rien ne se produit, au sens usuel) ou tentent d'étirer, de détourner une échelle temporelle narrative (réception ludique, " $[\ldots]$ the reconstitution by the spectator of the generating principles of the film [...]», James, p. 260). Ces œuvres impliquent donc intrinsèquement un nouveau mode d'écoute ou de visionnement où la mémoire et l'anticipation sont d'abord sollicitées d'une manière inédite (Fracture ou (nostalgia)), avant d'être, à la limite, disqualifiées (Drift Study ou Empire).

Le cinéma structurel et les compositions des répétitifs américains se sont démarqués des mouvements d'avant-garde qui les précédaient (le cinéma underground et la musique sérielle) par une exploration de limites de la logique formelle de leur médiums respectifs. Conséquemment, leur démarche peut être vue 
comme un retour sur les origines du médium ${ }^{9}$, impliquant une redéfinition du mode de réception par le spectateur. Un examen des films a pu révéler qu'à des problématiques communes aux deux arts voisins, les artistes ont apporté des «solutions» semblables.

En concentrant exclusivement notre analyse sur les répétitifs américains, nous avons volontairement tenu sous silence tout un pan de la musique contemporaine. Il aurait été intéressant, par exemple, de comparer le cinéma underground avec le rock underground. Deux notions très galvaudées mais qui, si l'on y regardait de plus près, pourraient aussi s'avérer révélatrices de similarités frappantes entre modes de production ou de réception culturels. Je pense en particulier à des groupes américains comme le Velvet Underground ou les Mothers of Invention, ou européens, comme Can, Faust ou Roxy Music. Ces groupes, tout en proposant une démarche assez stricte, explorèrent beaucoup plus que les compositeurs répétitifs les possibilités de l'improvisation et de l'utilisation de textes chantés ou récités.

\section{Université de Montréal}

\section{NOTES}

1 À ce sujet, voir entre autres: Ivanka Stoianova, "Musique répétitive», Musique en jeu, n" 26 (1977), p. 64-74 et Timothy A. Johnson, "Minimalism: Esthetic, Style, or Technique?", Musical Quarterly, vol. 78, n"4 (1994), p. 742-773. On peut aussi retrouver les différentes réponses qu'apportent les répétitifs américains, et d'autres compositeurs, à ce point précis d'une définition de leur art dans Geoff Smith et Nicola Walker Smith, American Originals. Interviews With 25 Contemporary Composers (London: Faber and Faber, 1994).

2 En ce qui concerne les reproches faits à Sitney, voir Dominique Noguez, Une renaissance du cinéma. Le cinéma underground américain (Paris: Klincksieck, 1985 , p. 346-351).

3 À propos de l'cuvre musicale méconnue de Tony Conrad, voir John Corbett, "Minimal Compact. Tony Conrad", The Wire, n" 132 (1995), p. 34-35 et Tony Conrad, "Inside the Dream Syndicate», Film Culture, n" 41 (1966), p. 5-8.

4 Sur la musique Fluxus, voir Barbara Moore, "Hocus Fluxus", Ear Magazine, vol. 15, n" 3 (1990), p. 20-24 et Eckart Rahn, "Musique sans musique", Musique en jeu, n"1 (1970), p. 26-33.

5 Cité par Caux dans les notes de pochette du disque.

6 On reconnaitra ici une structure semblable à celle du rondo, dans un tout autre paradigme (musique classique). Comparez par exemple, In $C$ de Riley (1964) et le rondo de la sonate Waldstein, op. 53, de Beethoven. 
7 La nationalité des Rimmer, McLaren et Snow est un autre exemple à rajouter au grief d'américano-centrisme ou d'hégémonie new-yorkaise implicite que Maciunas adresse à Sitney (Dominique Noguez, Une renaissance du cinéma. Le cinéma underground américain, Paris: Klincksieck, 1985, p. 346-351): Snow, artiste torontois, a été étroitement lié au milieu artistique new-yorkais, comme en témoignent l'impact d'un film comme Wavelength (1967), ou à un niveau plus anecdotique, sa participation à titre de narrateur au film (nostalgia) de Hollis Frampton (1971); McLaren, lui, débarque à New York en 1940 avec la ferme ambition de "[...] faire des films abstraits pour interpréter l'esprit de la musique", avant d'y être recruté par Grierson pour fonder le département d'animation de l'ONF (Léo Bonneville, "Norman McLaren au fil de ses films (interview)", Séquences, n` 82, 1975, p. 11).

8 Voici, par exemple, les élans que Pas de deux insuffle à Dominique Noguez: "[...] le spectateur, pareil au myste des mystères antiques, est témoin de l'épiphanie superbe de ce que, s'il était d'aventure platonicien, sans peine il pourrait identifier comme le Beau en soi, ou la Grâce en soi " (Éloge du cinéma expérimental, Paris: Centre national d'art et de culture Georges Pompidou, 1979, p. 39). Le film de McLaren emporte aussi Patrick Scupp: "Ce Pas de detax est non seulement une ouvre d'amour et d'intelligence, c'esr aussi une de celles par lesquelles on accède au plan divin, supérieur, que l'on pressent, mais que l'on est bien incapable d'atteindre " " $\mathrm{Du}$ rychme et des couleurs", Séquences, n" 82, 1975, p. 104).

9 On pourrait concevoir Empire comme une extension de L'Arrivée d'un train (1895) des frères Lumière, ou Pas de detix comme une extrapolation des chronophotographies de Marey (1883-1888), surtour si l'on considère que "[...] la musique a été rajoutée après le tournage" (Léo Bonneville, "Norman McLaren au fil de ses films (interview)", Séquences, n” 82, 1975, p. 83). Sur ce curieux retour aux origines, voir Tom Gunning, "An Unseen Energy Swallows Space: The Space in Early Film and Its Relation to American Avant-Garde Film* (Film Before Griffith, J. Fell (direction), Berkeley: University of California Press, 1983, p. 355-366) et Noel Burch, "Primitivism and the Avant-Gardes: A Dialectical Approach" (Narrative, Apparatus, Ideology, P. Rosen (direction), New York: Columbia University Press, 1986, p. 483505).

\section{OUVRAGES CITÉS}

Bonneville, Léo et al. "Norman McLaren au fil de ses films (interview)". Séquences, n" 82 (1975), p. 7-93.

Caux, Daniel. "Notes de pochette». Four Organs / Phase Patterns (Steve Reich). Shandar, 83.511.

Caux, Daniel. "Cette musique que l'on dit "répétitive" ". Musique en jeu, n" 26 (1977), p. 81-86.

Chion, Michel et Guy Reibel. Les Musiques électroacoustiques. Aix-en-Provence: Édisud, 1976.

Cope, David H. New Directions in Music. Dubuque: W.M.C. Brown Co., 1976.

Glass, Philip. "Notes de pochette». Music in Twlelue Parts. Parts I\&2 (Philip Glass). Virgin, CA2010, 1974.

Hébert, Pierre. "Procès d'une collection". Objectif 65, n" 32 (1965), p. 3-16.

James, David E. Allegories of Cinema. American Film in the Sixties. Princeton: Princeton University Press, 1989.

Koch, Stephen. Hyperstar. Andy Warhol, son monde et ses films. Paris: Du Chêne, 1974. 
Noguez, Dominique. Éloge du cinéma expérimental. Paris: Centre national d'art et de culture Georges Pompidou, 1979.

Noguez, Dominique. Une renaissance du cinéma. Le cinéma underground américain. Paris: Klincksieck, 1985 .

Nyman, Michael. "Notes de pochette". Voices and Instruments (Jan Steele et John Cage). Obscure, n" 5, 1975.

Nyman, Michael. Experimental Music. Cage and Beyond. New York: Schirmer Books, 1974.

Schupp, Patrick. "Du rythme et des couleurs». Séquences, n“ 82 (1975), p. 99-104.

Simon, L. "Music and Film: An Interview with Philip Glass». Millenium, n" 13 (1983-84), p. 133-139.

Tordjman, Gilles. "Perpetuum mobile. Philip Glass». Les Inrockuptibles, n” 52 (1994), p. 98-102.

Wentz, Brooke. "La Monte Young. The True Minimalist». OP, n" Y (1984), p. 4849. 